\title{
RESEARCH
}

Open Access

\section{Development, characterization, and hematopoietic differentiation of Griscelli syndrome type 2 induced pluripotent stem cells}

Gülen Güney-Esken ${ }^{1,2}$, Özgür Doğuş Erol ${ }^{1,2}$, Burcu Pervin ${ }^{1,2}$, Gülben Gürhan Sevinç ${ }^{3}$, Tamer Önder $^{3}$, Elif Bilgiç ${ }^{4}$, Petek Korkusuz ${ }^{1,2,4}$, Ayşen Günel-Özcan ${ }^{1,2}$, Duygu Uçkan-Çetinkayaa ${ }^{1,2,5}$ and Fatima Aerts-Kaya ${ }^{1,2,6^{*}}$ (D)

\begin{abstract}
Background: Griscelli syndrome type 2 (GS-2) is a rare, autosomal recessive immune deficiency syndrome caused by a mutation in the RAB27A gene, which results in the absence of a protein involved in vesicle trafficking and consequent loss of function of in particular cytotoxic T and NK cells. Induced pluripotent stem cells (iPSC) express genes associated with pluripotency, have the capacity for infinite expansion, and can differentiate into cells from all three germ layers. They can be induced using integrative or non-integrative systems for transfer of the Oct4, Sox2, Klf4, and CMyc (OSKM) transcription factors. To better understand the pathophysiology of GS-2 and to test novel treatment options, there is a need for an in vitro model of GS-2.

Methods: Here, we generated iPSCs from 3 different GS-2 patients using lentiviral vectors. The iPSCs were characterized using flow cytometry and RT-PCR and tested for the expression of pluripotency markers. In vivo differentiation to cells from all three germlines was tested using a teratoma assay. In vitro differentiation of GS-2 iPSCs into hematopoietic stem and progenitor cells was done using Op9 feeder layers and specified media.

Results: All GS-2 iPSC clones displayed a normal karyotype (46XX or 46XY) and were shown to express the same RAB27A gene mutation that was present in the original somatic donor cells. GS-2 iPSCs expressed SSEA1, SSEA4, TRA-1-60, TRA-1-81, and OCT4 proteins, and SOX2, NANOG, and OCT4 expression were confirmed by RT-PCR. Differentiation capacity into cells from all three germ layers was confirmed using the teratoma assay. GS-2 iPSCs showed the capacity to differentiate into cells of the hematopoietic lineage.

Conclusions: Using the lentiviral transfer of OSKM, we were able to generate different iPSC clones from 3 GS-2 patients. These cells can be used in future studies for the development of novel treatment options and to study the pathophysiology of GS-2 disease.
\end{abstract}

Keywords: Griscelli syndrome type 2, Bone marrow, Mesenchymal stromal cells, Hematopoietic stem cells, Induced pluripotent stem cells

\footnotetext{
* Correspondence: fatimaaerts@yahoo.com

'Graduate School of Health Sciences, Department of Stem Cell Sciences, Hacettepe University, Sihhiye, 06100 Ankara, Turkey

${ }^{2}$ Center for Stem Cell Research and Development (PEDI-STEM), Hacettepe University, Sihhiye, 06100 Ankara, Turkey

Full list of author information is available at the end of the article
}

(c) The Author(s). 2021 Open Access This article is licensed under a Creative Commons Attribution 4.0 International License, which permits use, sharing, adaptation, distribution and reproduction in any medium or format, as long as you give appropriate credit to the original author(s) and the source, provide a link to the Creative Commons licence, and indicate if changes were made. The images or other third party material in this article are included in the article's Creative Commons licence, unless indicated otherwise in a credit line to the material. If material is not included in the article's Creative Commons licence and your intended use is not permitted by statutory regulation or exceeds the permitted use, you will need to obtain permission directly from the copyright holder. To view a copy of this licence, visit http://creativecommons.org/licenses/by/4.0/ The Creative Commons Public Domain Dedication waiver (http://creativecommons.org/publicdomain/zero/1.0/) applies to the data made available in this article, unless otherwise stated in a credit line to the data. 


\section{Background}

Griscelli syndrome type 2 (GS-2) is a rare, inherited, autosomal recessive disease with a frequency of $<1 / 1$, 000,000 in neonates and has been shown to occur more often in Middle Eastern countries, such as Turkey, due to the increased rate of consanguineous marriages [1]. The disease is characterized by partial albinism (silvercolored hair, eyebrows, and eyelashes), hepatosplenomegaly, pancytopenia, immune deficiency, and neurological dysfunction [2]. In GS-2, RAB27A mutations cause the malfunction of a small GTPase, which plays an important role in vesicular fusion and cellular trafficking [2]. The RAB27A protein is responsible for the peripheral distribution of melanosomes in melanocytes and exocytosis of cytotoxic granules in the cytosol of cytotoxic $\mathrm{T}$ cells (CTL) and natural killer (NK) cells. In addition, RAB27A plays an important role in the binding of cytosolic granules to the cell membrane after $\mathrm{T}$ cell receptor signal activation [3-5]. GS-2 patients may also display a decrease in NK cell cytotoxicity resulting in impaired and late hypersensitivity [5]. Although in most cases granulocyte and lymphocyte counts are within normal limits, immunoglobulin levels may be decreased or increased. The only curative treatment for GS-2 is hematopoietic stem cell (HSC) transplantation [1, 6-8]. However, in the absence of human leukocyte antigen (HLA)-compatible donors, no other curative treatment options are available, and therefore, new treatment strategies, such as gene therapy, should be explored.

Via exogenous delivery of the transcription factors Oct4, Sox2, Klf4, and cMyc (OSKM), somatic cells can be reprogrammed into induced pluripotent stem cells (iPSCs) [9], which express typical pluripotency markers, such as SSEA-4, TRA-1-60, TRA-1-81, and alkaline phosphatase, and display three germ layer differentiation potential [9]. Although iPSC lines have been developed from various cell types [10], reprogramming efficiency and differentiation capacity of the iPSCs may vary according to cell source or type [11]. Especially in cases where sufficient cells cannot be obtained due to the rarity of the disorder, iPSC models have shown great potential in providing research material to develop new treatments or diagnostics, but also to study the pathophysiology of the diseases $[10,12]$. iPSC lines have been generated for a variety of diseases, including childhood hereditary diseases, and are now deposited in and made available through international iPSC biobanks [13-15]. In addition to disease modeling, iPSCs also enable the development and testing of new treatment modalities, such as gene editing.

Here, we aimed to generate iPSC lines from two different somatic cell types, i.e., mononuclear cells (MNCs) and multipotent mesenchymal stromal/stem cells (MSCs), that were obtained from 3 different GS-2 patients. We intended to generate an in vitro model reminiscent of GS-2 that (1) can be used as a platform to test new treatment strategies, such as gene therapy, and (2) will allow us to obtain a better understanding of the direct effects of the RAB27A mutation on differentiation and function and behavior of hematopoietic cells.

\section{Materials and methods}

\section{Healthy donor and GS-2 bone marrow samples}

Bone marrow (BM) samples of $1-5 \mathrm{~mL}$ were aspirated from GS-2 patients $(n=3)$ and healthy donors $(n=7)$ for diagnostic and/or transplantation purposes, after obtaining written informed consent from each donor. Procedures and sampling were approved by the Hacettepe University Non-Interventional Ethical Committee (GO14/424-19).

\section{Mesenchymal stromal cell isolation and culture}

$\mathrm{BM}-\mathrm{MNCs}$ were isolated using gradient centrifugation (Biocoll, Biochrom, L6115). MNCs were cultured in DMF10 medium, consisting of a mixture of $60 \%$ DMEM-LG (Life Technologies, 31885-049) and 40\% MCDB-201 (Sigma-Aldrich, M6770), supplemented with $10 \%$ fetal bovine serum (FBS, Life Technologies, 10270106), 1\% L-glutamine (Sigma-Aldrich, G3126), and 1\% penicillin-streptomycin (Biochrom, 02A2213). The adherent fraction consisting of mesenchymal stromal cells was expanded at $37^{\circ} \mathrm{C}$ and $5 \% \mathrm{CO}_{2}$, with medium replacements every 3-4 days. Confluent cultures were passaged with $0.25 \%$ trypsin/1 mM EDTA (Life Technologies, 27250-018).

\section{Characterization of MSCs}

For adipogenic differentiation, MSCs were cultured in adipogenic differentiation medium, consisting of DMEM-LG, 10\% FBS, $1 \mu$ M dexamethasone (Sigma-Aldrich D2915), $60 \mu \mathrm{M}$ indometacin (Sigma-Aldrich, 17378), $500 \mu \mathrm{M}$ 3-isobutyl-1-methylxanthine (Sigma-Aldrich, I5879), and $5 \mu \mathrm{g} / \mathrm{mL}$ insulin. After 3 weeks, cells were stained with $2 \mathrm{mg} / \mathrm{mL}$ Oil Red O (ORO, SigmaAldrich O0625). ORO dye was extracted from the cells using \%2 Igepal (NP40, Sigma-Aldrich, I8896) and measured at $496 \mathrm{~nm}$ (Tecan Sunrise microplate reader), as previously described [16]. For osteogenic differentiation, MSCs were cultured in an osteogenic differentiation medium, consisting of DMEM-LG, 10\% FBS, $100 \mathrm{nM}$ dexamethasone, $10 \mathrm{mM}$ beta-glycerophosphate (SigmaAldrich, G9422), and $0.2 \mathrm{mM}$ L-ascorbic acid (Sigma-Aldrich, A92902). To confirm osteogenic differentiation, cells were stained with Alizarin Red S (ARS). Calcium levels were measured using the Quantichrom Calcium Analysis kit (BioAssay Systems, DICA-500), as previously described [16]. 


\section{iPSC generation and expansion}

To establish iPSC lines, BM-MNCs or MSCs from three GS-2 patients and one healthy donor were transduced for 2 consecutive days with bicystronic (pSIN4-CMVK2M, Addgene, 21164; pSIN4-EF1 $\alpha$-O2S, Addgene, 21162) or polycystronic lentiviral vectors (pRRL-PPTSF-OSKM-IRES-idTomPRE), kindly provided by Prof. Dr. Axel Schambach, Hannover Medical School, Germany [17], carrying the reprogramming factors Oct3/4, Sox2, Klf4, and c-Myc. After transduction, MSCs were cultured in DMF10 and MNCs in StemMACS HSC expansion medium (Miltenyi, 130-100-463) supplemented with StemMACS HSC cytokine cocktail (STF, Stem Cell Factor/Thrombopoietin/Flt3-ligand, Miltenyi, 130-100-843) for 7 days. Transduced cells were plated on Matrigel-coated dishes (Corning, 354277), and the culture medium was replaced by StemMACS iPSBrew XF culture medium (iPS-Brew, Miltenyi, 130-107086) with $20 \mathrm{ng} / \mathrm{mL}$ basic fibroblast growth factor (bFGF, Immunotools, 11343627). Colonies were picked around day 18 after reprogramming, disrupted by microdissection, and re-plated in iPS-Brew/bFGF supplemented with $10 \mu \mathrm{M}$ Rock inhibitor Y27632 (Miltenyi, 130-103-922).

\section{Immunophenotyping of MSCs and iPSCs}

MSCs were stained with antibodies against CD29 (BioLegend, 303008), CD44 (BioLegend, 338804), CD73 (BioLegend, 344006), CD90 (BioLegend, 328108), CD105 (eBioscience, 12-1057-41), CD166 (BioLegend, 343904), CD34 (eBioscience, 17-0349-42), CD31 (BD Biosciences, 555445), and HLA-DR (BD Biosciences, 307606) in PBN consisting of phosphate-buffered saline (DPBS 1X, Gibco, 14190144), 0.5\% bovine serum albumin (BSA, Sigma, A4503), and $0.05 \%$ sodium azide $\left(\mathrm{NaN}_{3}\right.$, Merck, $822335)$ supplemented with $2 \%$ human AB serum. MSCs were assessed with a FACSARIA (Becton Dickinson) or BDAccuri (Becton Dickinson) and analyzed with the FACSDiva V6.12 or BD CSampler analysis software. For iPSC characterization, surface antigens were stained with antibodies against SSEA1 (Miltenyi, 130-104-937), SSEA4 (Miltenyi, 130-098-347), TRA-1-60 (Miltenyi, 130-100-347), and TRA-1-81 (Miltenyi, 130-101-410) in MACS Buffer (PBS, 0.5\% BSA and 2 mM EDTA). For intracellular staining, cells were permeabilized with $0.2 \%$ Triton (Sigma-Aldrich, X100)/0.2\% Tween-20 (SigmaAldrich, P1379) and stained with anti-Oct3/4 (Miltenyi, 130-105-555) antibody. iPSCs were washed twice with MACS buffer and measured with a flow cytometer.

\section{RNA isolation, CDNA synthesis, and quantitative real-time PCR}

Total RNA was extracted from iPSCs using Qiazol lysis reagent (Qiagen, 79306) and RNeasy plus Mini Kit
(Qiagen, 217004). For cDNA synthesis, $1.3 \mu \mathrm{g}$ of total RNA was used, and cDNA was synthesized using the Transcriptor High Fidelity cDNA Synthesis kit (Roche, 05081963001). RT-PCR assays were performed with the Lightcycler 480 probes Master kit (Roche, 04 707494 001) using a LightCycler 480 II (Roche). Expression of OCT4, SOX2, and NANOG pluripotency genes was normalized to the expression of the $B 2 M$ gene, and relative gene expression was calculated using the Livak method [18]. Used primer sequences are shown in Table S1.

\section{Immunofluorescent labeling of iPSCs}

To confirm the expression of pluripotency proteins in selected iPSC clones, clones were fixed with $4 \%$ paraformaldehyde and permeabilized with $0.2 \%$ Triton X100+ $0.2 \%$ Tween-20 in PBS for $20 \mathrm{~min}$, at room temperature. Cells were blocked with a blocking buffer consisting of $\mathrm{PBN}, 1 \% \mathrm{AB}$ serum, and $1 \%$ mouse serum. iPSCs were stained with the following primary antibodies: rabbitanti-human OCT3/4 (Molecular Probes, A24867), mouse-anti-human SSEA4 (Molecular Probes, A24866), mouse-anti-human TRA-1-60 (Molecular Probes, A24868), rabbit-anti-OCT4A (Cell Signaling Technology, 2840), rat-anti-SOX2 (Cell Signaling Technology, 3579), rabbit-anti-NANOG (Cell Signaling Technology, 4903), rabbit-anti-c-MYC (Cell Signaling Technology, 5605), and rabbit-anti-LIN28A (Cell Signaling Technology, 3695). Incubations were performed for $2 \mathrm{~h}$ at room temperature. Cells were with PBN and then incubated with one of the following secondary antibodies: goatanti-rabbit IgG-Alexa Fluor 488 (Thermo Fisher Scientific, A11008), goat-anti-mouse IgG-Alexa Fluor 488 (Thermo Fisher Scientific, A11029), anti-rat IgG-Alexa Fluor 488 (Molecular Probes, A24876), anti-mouse IgGAlexa Fluor 488 (Molecular Probes, A24877), goat-antirabbit IgG-Alexa Fluor 568 (Abcam, ab150077), or ratanti-mouse IgG-FITC (BD Biosciences, 553443). Incubations were performed for $1 \mathrm{~h}$ at room temperature, in the dark. Cells were stained with DAPI (300 nM, SigmaAldrich, D9542) and analyzed using an Olympus fluorescent microscope.

\section{Mutation analysis of patient cells before reprogramming} $R A B 27 A$ gene mutations were determined in the material of all three GS-2 patients before and after reprogramming. Selected iPSC clones were expanded and all coding domains, as well as exon-intron intersection domains were assessed by next-generation sequencing with a Miseq device (Illumina) and analyzed using the Miseq Reporter (Illumina) software. Aligned "bam" files were analyzed with the IGV 2.3 (Broad Institute) software. 


\section{Karyotyping of iPSCs}

For karyotyping, 3-4 drops of Colcemid were added to the culture and incubated for $3 \mathrm{~h}$. iPSC cultures were trypsinized, and hypotonic salt solution was added to the cell pellets. Cells were prefixed with 5-6 drops of fixative, centrifuged, and resuspended in $5 \mathrm{~mL}$ fixative. Cells were washed twice, transferred to slides, and stained with Giemsa. A minimum of 10 metaphases was captured and analyzed.

\section{In vivo teratoma formation and characterization}

$1 \times 10^{6}$ iPSCs from different clones derived from both healthy donors and GS-2 patients were resuspended in $100 \mu \mathrm{L}$ Matrigel and injected directly in the left and right gastrocnemius muscle of Balb/c-Rag2 ${ }^{-/-}$mice $(n=$ 10), kindly provided by Prof. Dr. Gerard Wagemaker, Erasmus Medical Center Rotterdam, The Netherlands [19], after pretreatment with $25 \mathrm{mg} / \mathrm{kg}$ Busulfan (Busilfex, Abdi İbrahim). All animal experiments were approved by the local Hacettepe University animal experiments ethical committee (2014/46-03 and 2015/ 68-10) and performed at the Hacettepe University Laboratory Animals Research and Application Centre. Teratomas were removed after 6-8 weeks of transplantation for histological analysis and infiltrated with paraffin following immersion fixation in $10 \%$ buffered formalin, dehydration in graded series of ethanol, and clearing in xylene. Three to five-micrometer-thick hematoxylin/eosin-stained sections were evaluated under a light microscope (Leica DM6B) with an attached digital camera in order to confirm the presence of three germ layer differentiation using the LASX software.

\section{In vitro hematopoietic differentiation of iPSCs}

$1 \times 10^{6}$ healthy donor or GS-2 iPSCs were co-cultured with Op9 stromal cells (kindly provided by Prof. Dr. Juan Carlos Zúñiga-Pflücker, Sunnybrook Research Institute, Toronto, Canada [20]) in different HSC differentiation media for 8 days. A total of 7 clones (2 from healthy donor iPSCs, 5 derived from 3 different GS-2 patients) were tested for HSC differentiation potential. HSC differentiation media consisted of serum-free StemMACS HSC expansion medium with $1 \times$ hematopoietic expansion cocktail (STF: SCF, TPO, Flt3-ligand), 50 ng/ mL BMP4 (Bone Morphogenic Protein 4 (R\&D Systems, 314-BP-050), $5 \mu \mathrm{M}$ ATRA (all-trans retinoic acid, Sigma-Aldrich, R2625) and $1 \mu \mathrm{M}$ dexamethasone. Differentiated CD34+ HSC were further expanded with StemMACS HSC expansion medium with $1 \times$ STF for 1-2 weeks. Immunophenotype of the differentiated cells was measured using anti-CD43 (BioLegend, 343206), antiCD45 (BD Biosciences, 560976), anti-CD34 (eBioscience, 17-0349-42), and anti-CD38 (BD Biosciences, 555459) antibodies. To assess the colony-forming capacity of
HSCs differentiated from iPSC, the cells were cultured in Methocult H4434 classic (Stem Cell Technologies, 04444) for 10-14 days. After counting, colonies were picked and stained with anti-CD45, anti-CD16 (BioLegend, 302012), anti-CD14 (BD Biosciences, 555399), and anti-CD33 (BioLegend, 366608) to confirm myeloid differentiation.

\section{Transplantation and engraftment of iPSC-derived HSCs}

To test the quality of HSCs derived from a healthy donor and GS-2 iPSCs, a total of 15 mice were transplanted with $5 \times 10^{5}-1 \times 10^{6}$ HSCs derived from healthy donor iPSCs ( $n=3$, control group) or GS-2 iPSCs $(n=12)$. The HSCs were cultured expanded as explained above, resuspended in $100 \mu \mathrm{L}$ PBS and transplanted i.v. into immune-deficient $\mathrm{Balb} / \mathrm{c} \mathrm{Rag}^{-/-}$mice after pretreatment with $25 \mathrm{mg} / \mathrm{mL}$ busulfan (BU). Mice were assessed for human engraftment by measuring the levels of anti-human CD45, CD10 (BD Biosciences, 347503), CD19 (BD Biosciences, 555413), CD3 (BD Biosciences, 555333), CD4 (BD Biosciences, 555346), and CD8 (BD Biosciences, 555369) in peripheral blood samples at months 1, 2, 3, and 6 after transplantation. Bone marrow and spleen cellularity and human engraftment were assessed following sacrifice of mice from each group at 3 or 6 months after transplantation.

\section{Statistical analysis}

Differences in gene expression levels between somatic cells before reprogramming and generated iPSC clones were determined after normalizing genes of interest with the reference gene. The standard deviation of the mean was calculated, and $T$ tests were performed using the Excel software program. $P$ values $<0.05$ were considered significant.

\section{Results}

Characteristics of GS-2 and healthy donor BM-MSCs

Microscopically, MSCs from healthy and GS-2 samples were similar and showed a spindle-shaped morphology (Fig. 1a). No significant differences were found in terms of differentiation capacity (Fig. 1a, Table S2). Both healthy and GS-2 MSCs were highly positive for specific MSC markers CD29, CD44, CD73, CD90, CD105, CD166, and HLA-DR and negative for the endothelial and hematopoietic markers CD31 and CD34 (Fig. 1b, Table S3).

\section{Lentiviral reprogramming of healthy and GS-2 MNCs and MSCs}

Eighteen days after reprogramming of healthy donor and GS-2 MNCs, using LV-SF-OSKM, formation of idTOMpositive iPSC-like colonies was observed (Fig. 2a). Passage 3 BM-MSCs from healthy $(n=1, \mathrm{GP})$ and GS-2 $(n=$ 


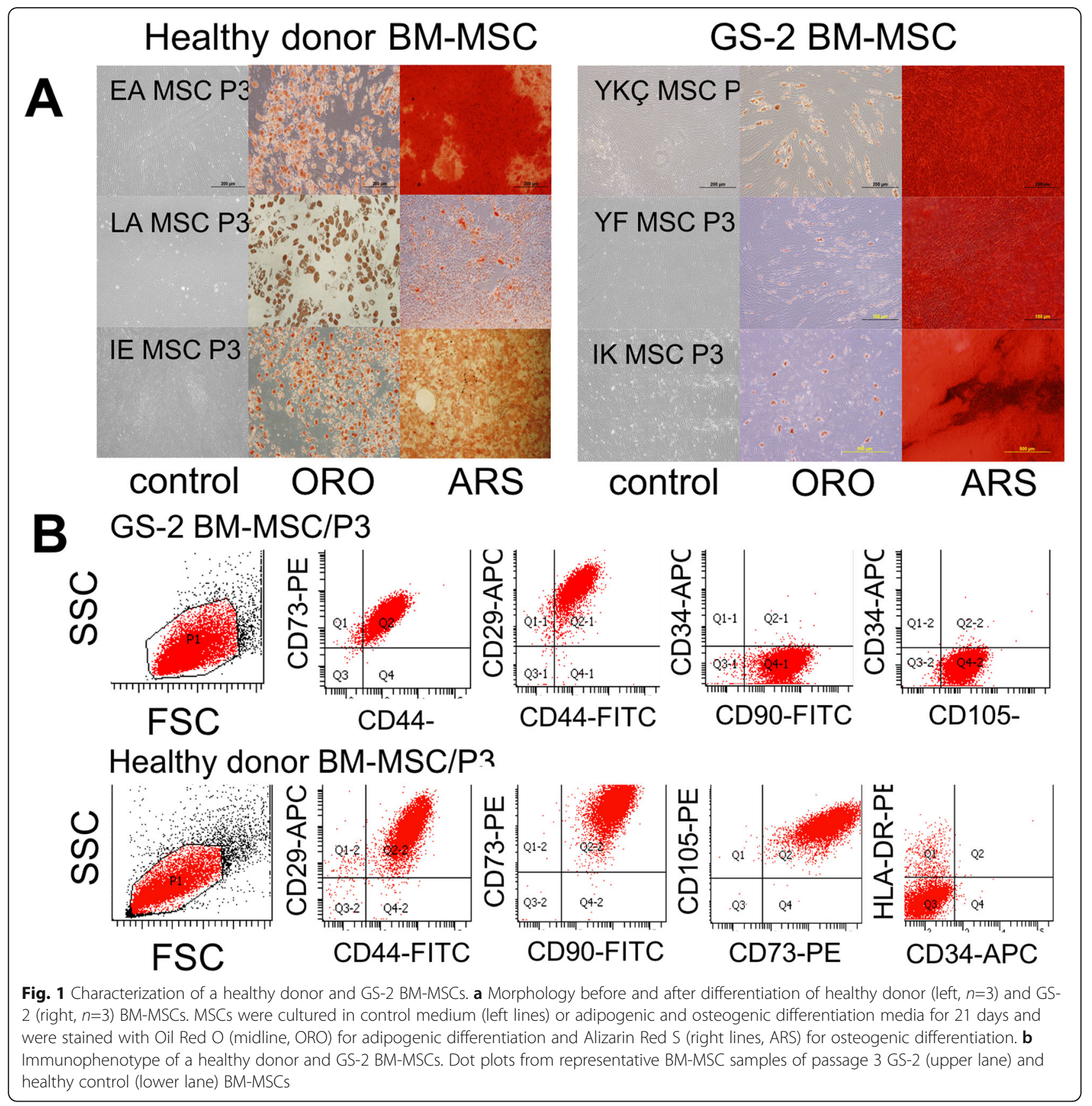

3; YF, IK, YKÇ) donors were reprogrammed using the bicistronic vector for YF and IK and the polycistronic vector for YKC, and colonies were collected between 18 and 21 days. At least 5 iPSC clones were generated for each sample, and the best clones were chosen for further use and characterization (Fig. 2b). iPSC clones derived from healthy and GS-2 samples maintained consistent morphology and growth rates for up to 12 passages.

\section{Characterization of healthy donor and GS-2 iPSC clones} iPSC clones generated from BM-MNCs expressed the pluripotency markers SSEA-4 and OCT3/4, but not the differentiation-related marker SSEA-1. MNC-derived GS-2 iPSC cells did not express MSC or hematopoietic surface markers (CD73, CD45), and CD90 expression was found to be low (Fig. 3a, top lane). BM-MSCderived healthy donor and GS-2 iPSC clones were highly positive for OCT3/4 and showed positivity for SSEA-1, SSEA-4, TRA-1-60, and TRA-1-81 (Fig. 3a, lower lane). Expression of LIN28, KLF4, c-MYC, OCT3/4, SOX2, and NANOG in healthy donor and GS-2 iPSC clones was further confirmed using immunofluorescent staining (Fig. 3b). All iPSC clones were analyzed for SOX2, NANOG, and OCT4 gene expression by RT-PCR. 


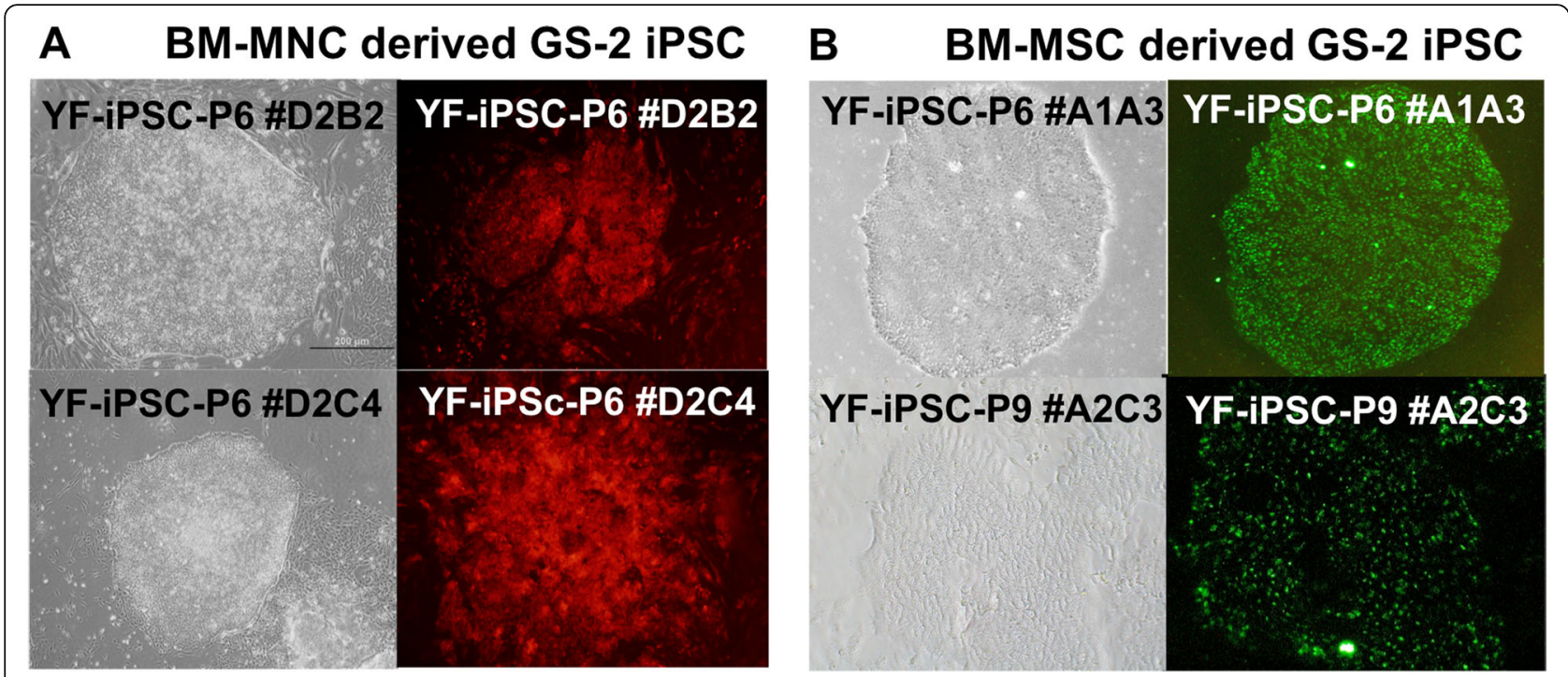

Fig. 2 Morphology of BM-MNC- and BM-MSC-derived iPSC clones. a Morphology and idTOM (red) expression of BM-MNC derived GS-2 iPSC clones YF \#D2B2 and \#D2C4. b Morphology and TRA-1-60 (green) expression of BM-MSC derived GS-2 iPS cell clones YF \#A1A3 and \#A2C3. Olympus inverted fluorescent microscope images, $\times 10$ magnification

Healthy and GS-2 BM-MSCs were used as negative controls. Healthy donor (LA) and GS-2 (YKÇ) BM-MSC SOX2, NANOG, and OCT4 gene expression was low in comparison with the iPSC clones. Increased gene expression of NANOG, OCT4, and SOX2 was detected in all iPSC clones tested (Fig. 3c), although not all clones showed a similar increase in gene expression.

Karyotyping of healthy and GS-2 iPSC clones showed normal karyotypes consisting of 46XX or 46XY chromosomes (Figure S1). Teratoma slides revealed the presence of mesodermal striated and smooth muscle, adipose tissue, cartilage, blood vessel, endodermal respiratory and gastrointestinal epithelia, and ectodermal epidermis and axons histologically (Fig. 4), confirming the potential for differentiation into three germ layers of these iPSC clones.

Mutation analysis of healthy and GS-2 iPSC clones confirmed the absence of $R A B 27 A$ mutations in healthy donor iPSCs and the presence of specific $R A B 27 A$ gene mutations at the same locations in the GS-2 iPSC clones and the original GS-2 samples (Table 1).

In vitro hematopoietic differentiation of healthy and GS-2 iPSCs

iPSCs were co-cultured on Op9 cells with hematopoietic differentiation medium 1 (HDM1: $\alpha M E M, 10 \%$ FBS-HI, $1 \%$ penicillin/ streptomycin, $1 \%$ L-glutamine, $5 \mathrm{mM} \beta$ mercaptoethanol, $4 \mathrm{mg} / \mathrm{mL}$ L-ascorbic acid, $10 \mu \mathrm{g} / \mathrm{mL}$ BMP-4) or hematopoietic differentiation medium 2 (HDM2: StemMACS HSC expansion medium, 1X STF, $10 \mu \mathrm{g} / \mathrm{mL}$ BMP-4). Within 4-5 days after co-culture, islands of hematopoiesis appeared in the cultures (Fig. 5a), and after 9 days of co-culture, cells were assessed for the presence of CD34, CD38, CD45, and/or CD43, a panhematopoietic marker, that has been shown to define the earliest hematopoietic progenitors in embryonic stem cell (ESC) cultures [21]. The results showed the presence of up to $14.3 \%$ CD34+ in serum-free HDM2 medium, despite considerable adipogenic differentiation of the stromal feeder layer Op9 cells. In contrast, significantly lower levels of CD34+ cells (4.3\%) were detected in co-cultures maintained in HDM1 where, interestingly, the morphology of the Op9 cells remained unchanged (Fig. 5b). Not all clones were tested for hematopoietic differentiation potential, but from the 7 clones (2 healthy donor iPSC clones, 5 GS-2 iPSC clones, derived from MNCs and MSCs) tested, with the exception of one GS-2 clone (YF \#A2C3), all clones showed expression of CD34+ after 9 days of co-culture with Op9 cells. This particular clone also showed much lower gene expression of NANOG and OCT4 (Fig. 3c) and was most likely only partially reprogrammed. Colony assays performed with iPSC-derived HSCs showed a preference for white colonies, which were confirmed to be mixed CFU-GM colonies expressing CD45 (91.6-92.3\%), CD33 (47.1-95.0\%), CD16 (9.09.1\%), and CD14 (12.4-17.5\%) markers (Fig. 5c). Optimization of the culture conditions by addition of ATRA and dexamethasone, in combination with further expansion of the iPSC-derived HSCs for an additional 21 days in presence of HSC expansion medium supplemented with 1X STF resulted in the expansion of considerable numbers of CD34+ cells, ranging from 40 to $80 \%$ CD34 after 14-21 days of expansion culture (Figure S2). When these cells were transplanted into $\operatorname{Rag} 2^{-1-}$ mice at a 


\section{A BM-MNC derived GS-2 iPSc YF \#D2B2}
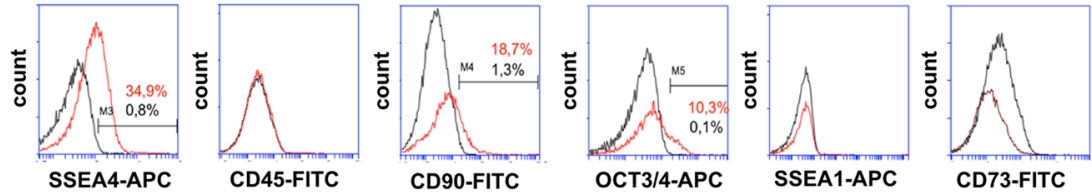

BM-MSC derived GS-2 iPSc YF \#A2C3

unstained control
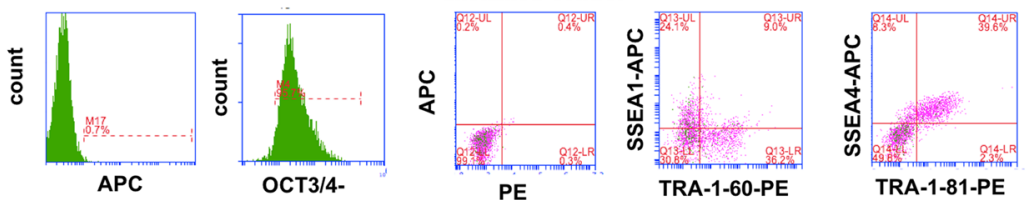

B Healthy donor GP iPSC P8 \#1
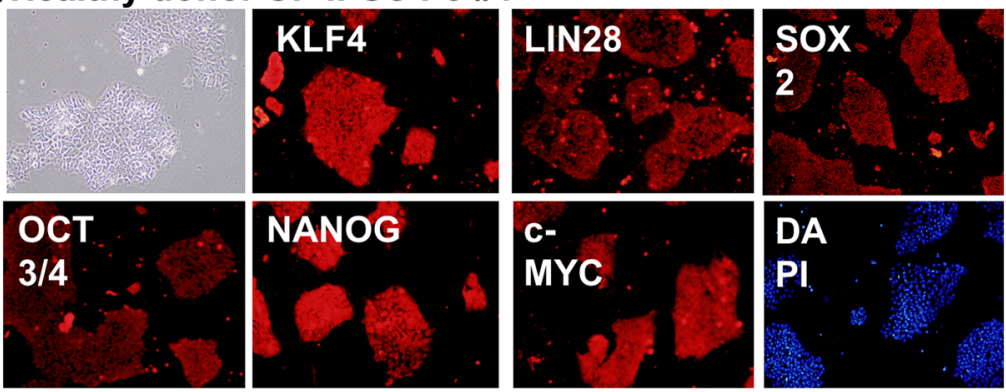

GS-2 YF iPSC P7 \#1
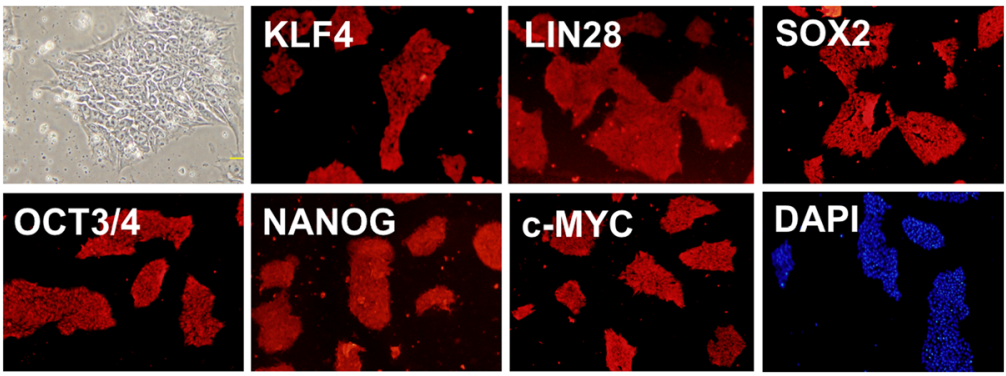

C 20,0

Expression of pluripotency genes

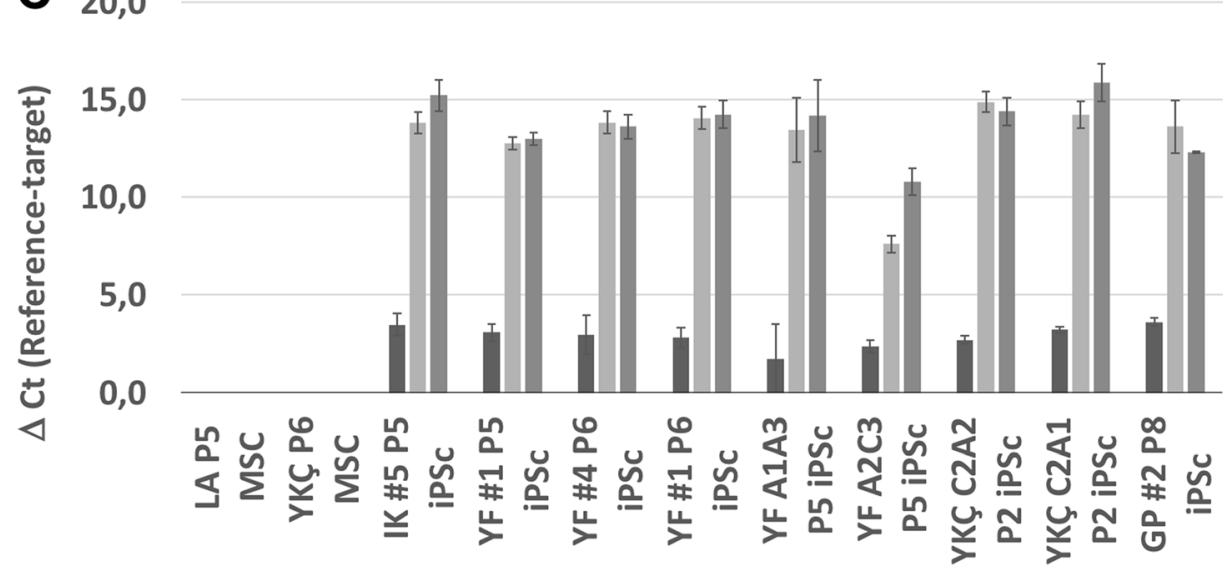


(See figure on previous page.)

Fig. 3 Immunophenotype of BM-MNC- and BM-MSC-derived iPSC clones. a Top lane: representative histograms of a GS-2 iPSC clone (YF \#D2B2) derived from BM-MNCs. Unstained controls are given as black lines and stained IPSC as red lines. Lower lane: representative histograms and dot plots from a BM-MSC-derived GS-2 iPSC clone (YF \#A2C3). b Healthy donor iPSC (top) and GS-2 iPSC (bottom) immunofluorescence images, representative samples. Both healthy donor and GS-2 iPSCs show bright expression of pluripotency markers. The nuclei were counterstained with DAPI. c Relative gene expression levels of SOX2, NANOG, and OCT4 normalized to B2M expression. Healthy (GP) and GS-2 (IK, YF, YKÇ) iPSC clones showed increased levels of SOX2, NANOG, OCT4 gene expression in comparison with healthy donor (LA) and GS-2 (YKÇ) BM-MSCs

(negative controls)

dose of $5 \times 10^{5}-10^{6}$ cells/mouse, engraftment was observed at 1 month after transplantation with the appearance of human cells in peripheral blood (PB) samples of mice (Fig. 6a). Maximal engraftment was observed at 2 months after transplantation, after which levels of human cells in murine PB steadily decreased. In addition to the presence of human leukocytes in $\mathrm{PB}$, the presence of CD34+ and CD45+ human hematopoietic cells was also detected in BM and spleen at 3 and 6 months after transplantation (Fig. 6b).

\section{Discussion}

GS-2 syndrome is a rare autosomal recessive immunodeficiency that can only be treated with HSC transplantation. The rarity of the disease makes it difficult to obtain sufficient cell numbers for research and to allow the development of new treatment strategies. The two GS-2 mouse models, i.e., the $\mathrm{C} 3 \mathrm{H} /$ $\mathrm{HeSn}-\mathrm{Rab} 27 \mathrm{a}^{\text {Ash}} / \mathrm{J}$ or "Ashen mouse" [22] and the C57BL/6J-Rab27a ${ }^{\text {Ash } / J ~(R a b 27 a ~}{ }^{-/-}$) mouse model created by backcrossing into C57BL/6J mice [23], both display an immune deficiency and HLH phenotype similar to GS-2 syndrome in humans. Nonetheless, an in vitro human GS-2 model is needed to develop and test new treatment strategies. Therefore, we aimed to generate several iPSC lines from GS-2 patients with different RAB27A mutations. Here, we show that iPSC clones derived from GS-2 MNCs and MSCs fulfill all the requirements of pluripotent stem cells, including the

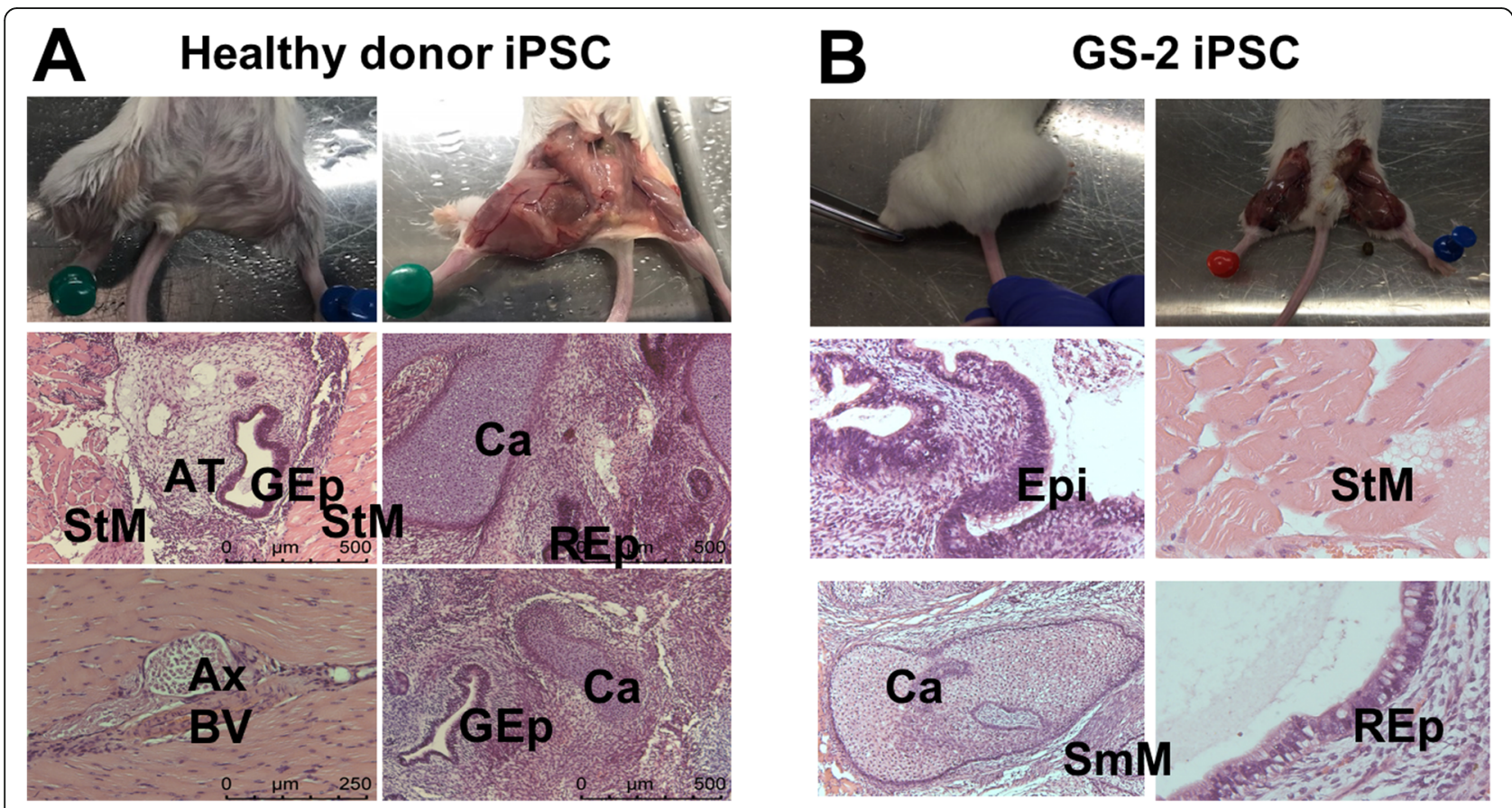

Fig. 4 Teratoma capacity of a healthy donor and GS-2 iPSCS. a $1 \times 10^{6}$ healthy donor iPSCs were injected intramuscularly in Rag ${ }^{-/-}$mice and followed for 6-8 weeks. Mice were sacrificed when palpable tumors appeared. Teratomas were fixed and stained with hematoxylin/eosin. Teratomas reveal the presence of mesodermal striated muscle (SM), adipose tissue (AT), cartilage (Ca), blood vessel (BV), endodermal respiratory (REp) and gastrointestinal (GEp) epithelium, and ectodermal axons (Ax). b $1 \times 10^{6} \mathrm{GS}-2$ iPSCs were injected intramuscularly in Rag2 $2^{-1-}$ mice. Teratomas reveal the presence of mesodermal striated muscle (StM) and smooth muscle (SmM), blood vessel (BV), cartilage (Ca), endodermal respiratory endothelium (RE), and ectodermal epidermis (Epi). Pictures from representative samples 
Table 1 RAB27A gene mutations present in healthy donor and GS-2 iPSC clones

\begin{tabular}{ll}
\hline iPSC clones & RAB27A (NM_004580) \\
\hline Healthy donors & \\
GP/P8/clone \#1 & Ok \\
GP/P8/clone \#2 & Ok \\
GS-2 patients IPSCs & \\
IK/P8/clone \#5 & c.514_518delCAAGC \%100 \\
IK/P6/clone \#2 & c.514_518delCAAGC \%100 \\
YF/P6/clone \#1 & c.148-149delAGinsC \%100 \\
YF/P6/clone \#4 & c.148-149delAGinsC \%100 \\
YF/P8/clone \#A1A3 & c.148-149delAGinsC \%100 \\
YF/P8/clone \#A2C3 & c.148-149delAGinsC \%100 \\
YKÇ/P6/clone \#C2A1 & c.148-149delAGinsC \%100 \\
YKÇ/P6/clone \#C2A2 & c.148-149delAGinsC \%100 \\
\hline
\end{tabular}

expression of pluripotency genes and differentiation into cells from all three germ cell layers.

In addition to the detailed characterization of GS-2 iPSCs, we also provide a detailed characterization of GS2 MSCs and compared the latter with healthy donor MSCs. In terms of "morphology" and immunophenotype, we found no differences between GS-2 and healthy donor MSCs. In addition, GS-2 MSC proliferation and differentiation capacity were similar to healthy donor MSCs. Thus, it appears that the RAB27A mutation does not negatively affect the overall proliferation or differentiation capacity of MSCs.

iPSC clones were successfully derived from both GS-2 MNCs and MSCs using lentiviral vectors, and no differences were observed in terms of morphology, immunophenotype, or expression of pluripotency genes in iPSCs derived from these two somatic cell sources. However, we did notice that using the same concentration of OSKM virus, MNCs showed a tendency for rapid reprogramming, whereas MSCs appeared to be more resistant to reprogramming, requiring a higher MOI. These data are in line with a study showing that cell type may affect reprogramming efficiency [24]. Similar to our results, Wang and colleagues showed that reprogramming of MNCs appeared to be more effective than reprogramming of MSCs [25].

Using different protocols to induce hematopoietic differentiation of the GS-2 iPSC clones, we found that MNC-derived iPSCs more rapidly differentiated towards HSCs, giving rise to up to $17 \% \mathrm{CD} 34+$ in co-cultures with Op9 cells. In contrast, HSC differentiation from iPSCs derived from GS-2 MSCs proved much more difficult, resulting in much lower percentages of CD34+ cells (ranging from 2 to 4\%). These data may indicate that the inherent, epigenetic memory of the iPSCs may influence both the efficacy of reprogramming, but also the differentiation capacity of these cells, at least in vitro. Accordingly, it has been shown previously that iPSCs may preserve their epigenetic memory and that tissue or somatic cell type-specific DNA methylation patterns may remain despite reprogramming, causing a relative preference of these cells to re-differentiate towards their cell type of origin rather than transdifferentiation into another lineage [26, 27].

Choi et al. co-cultured iPSCs with Op9 cells and found similar levels of CD34+ cells [28]. However, these levels leave room for improvement, and many groups are currently working on protocols to increase the efficacy and efficiency of HSC differentiation. For example, ATRA has been shown to increase hematopoietic differentiation potential [29]. In addition, certain molecules, such as WNT pathway inhibitors (CHIR99021) [30, 31], BMP4, and bFGF, have been reported to increase hematopoietic differentiation efficiency [32]. Using different combinations of these factors, we managed to increase the levels of CD34+ cells in vitro. Further culture modifications, including the use of gelatin-coated surfaces, different densities, and/or irradiation of Op9 cells, may further improve hematopoietic differentiation of iPSCs. Alternatively, Cerdan and colleagues performed HSC differentiation using embryonic body formation directly from ESCs and were able to obtain 90\% CD45+ cells [33]. iPSC-derived HSCs were transplanted in immunedeficient $\mathrm{Rag} 2^{-/-}$mice, and engraftment was followed for up to 6 months. Despite high levels of CD34+ cells obtained in in vitro cultures, we found that human chimerism after the initial engraftment rapidly decreased after 2 months of transplantation. This lack of long-term engraftment may have been caused by many factors, including prolonged in vitro expansion of the CD34+ cells, which may have depleted long-term repopulating cells. Furthermore, the cells were not further sorted or selected for CD34 positivity, and infused cell populations may not have been sufficient to sustain engraftment. Also, in contrast to the $\mathrm{Rag} 2^{-/-} / \mathrm{IL}_{2} \mathrm{R}_{\mathrm{\gamma c}}{ }^{-1-}$ mice, the Rag2 ${ }^{-/-}$mice that we used here are not the preferred humanized mouse model due to their remaining NK cell function. In addition, the low-dose preparatory regime used ( $25 \mathrm{mg} / \mathrm{kg}$ busulfan) may not have been sufficient to open up the bone marrow space and suppress remaining immunity. Therefore, engraftment might have been better or could have lasted longer in other humanized models, such as the NOD/SCID-IL2R ${ }^{-/-}$(NSG) mice and NSG mice transgenic for human hematopoietic growth factors [34]. Lastly, iPSC-derived CD34+ cells may show defects in homing and/or cannot be sustained/maintained in the bone marrow due to the absence of certain surface antigens, including adhesion molecules. It is furthermore conceivable that 


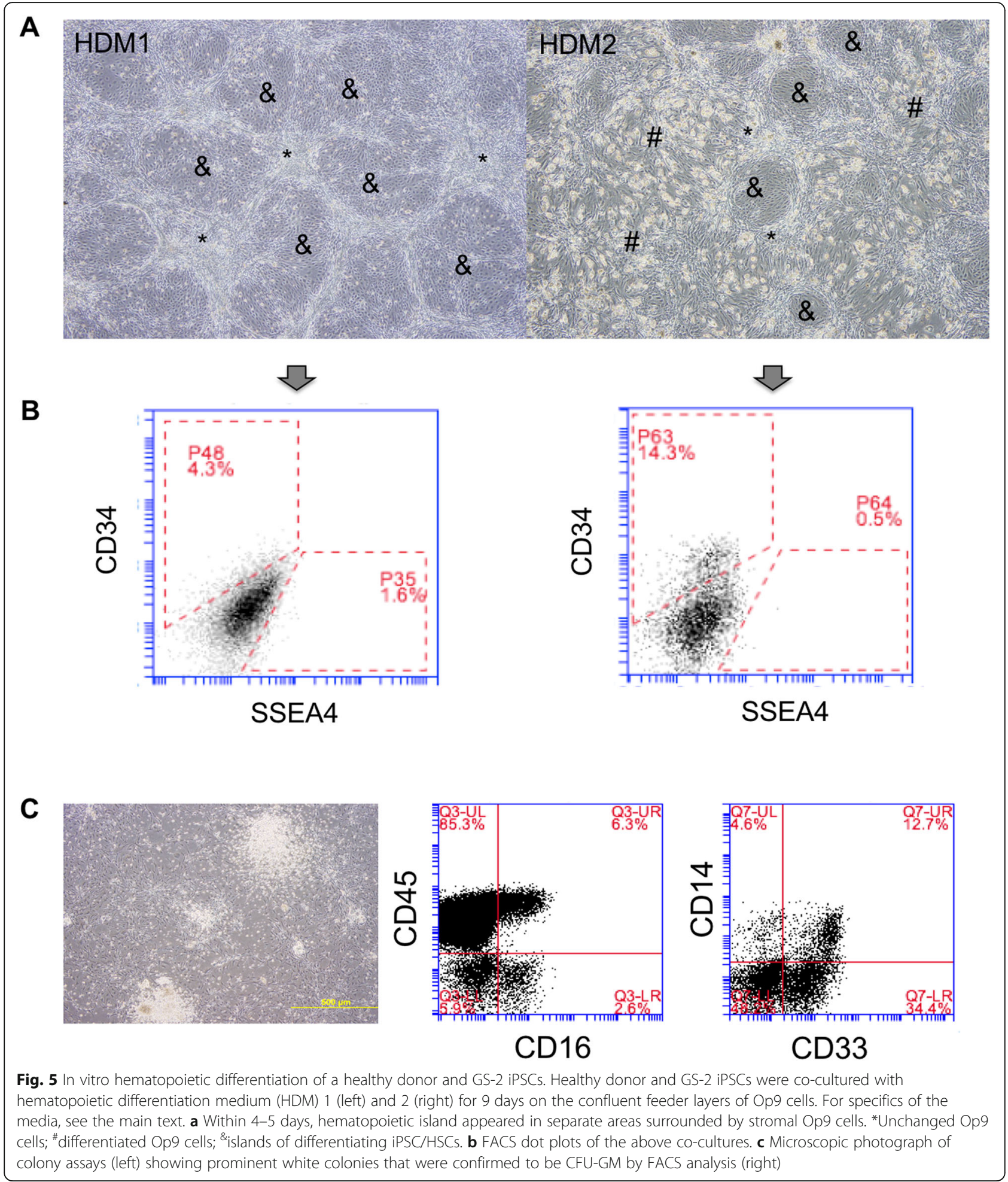

iPSC-derived CD34+ cells may not have sufficiently long telomeres to support long-term hematopoiesis. Since we cannot rule out that the observed shortterm engraftment was caused by the animal model, rather than by the transplanted iPSC-derived HSCs, all these factors should be further investigated. Nevertheless, the data are promising, and using modifications of the current protocols, both in vitro and in vivo, we believe that transplantation and long-term engraftment of iPSC-derived HSCs will be feasible. 


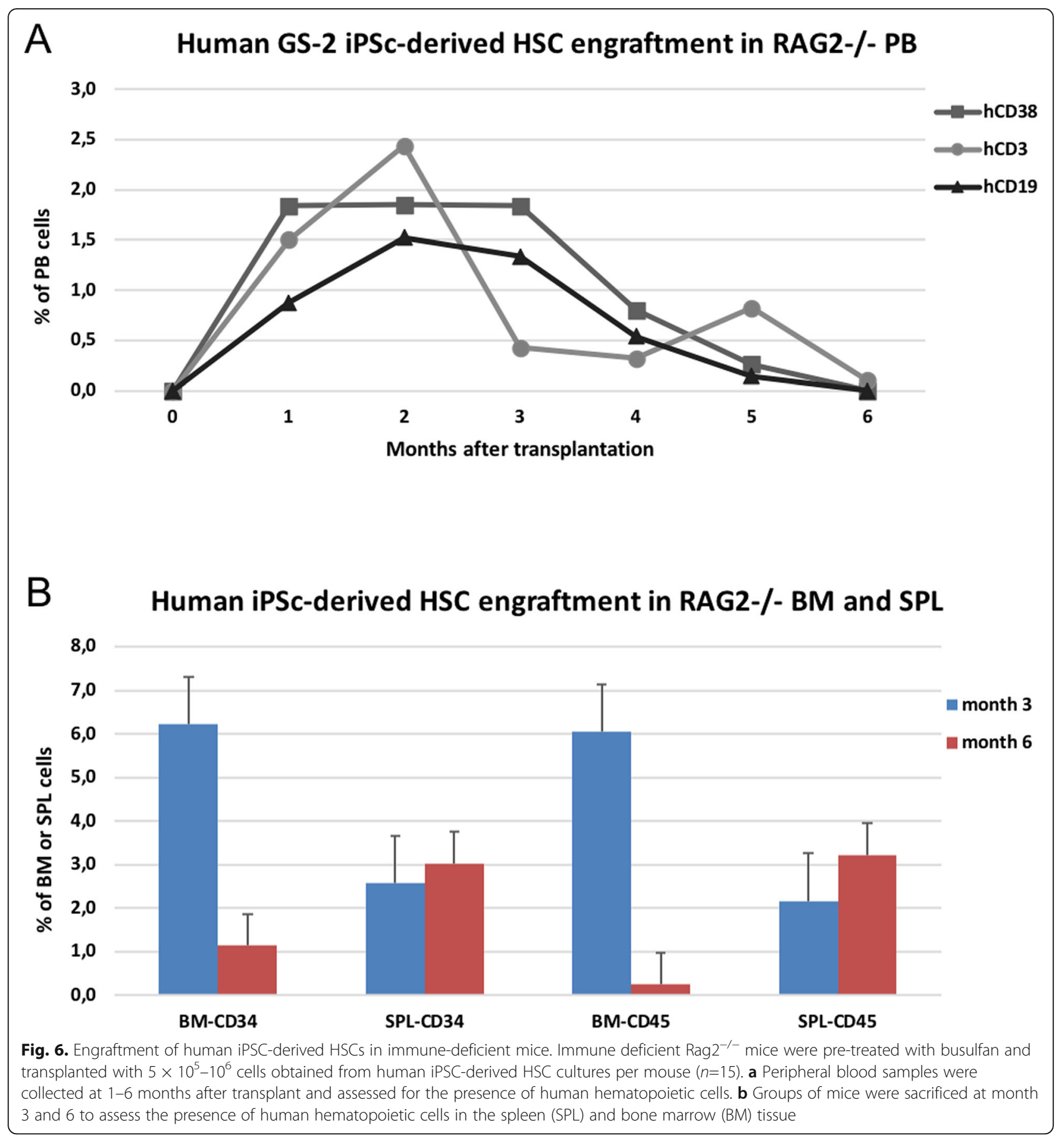

\section{Conclusions}

Here, we show that the iPSC clones derived from GS2 patients fulfill all requirements of true pluripotent stem cells, including the expression of pluripotency genes and the capacity for differentiation into cells of all three germlines. In addition, the ability of GS-2 iPSCs to differentiate in vitro into HSCs and allow, at least short-term, in vivo engraftment underlines the potential of these cells for the development of future treatment modalities. With further optimization of the protocols, these cells could serve as a model to investigate the effects of the RAB27A mutation on HSC differentiation and HSC potential and its role in the impairment of cytotoxic $\mathrm{T}$ cells and NK cells. Furthermore, this GS-2 iPSC model can be used to develop new lentiviral or CRISPR/Cas9-based gene therapy strategies for the treatment of Griscelli syndrome type 2 . 


\section{Abbreviations}

GS-2: Griscelli syndrome type 2; iPSCs: Induced pluripotent stem cells; OSKM: Oct4, Sox2, KIf4, and CMyc; CTL: Cytotoxic T cells; NK cells: Natural killer cells; HSCs: Hematopoietic stem cells; HLA: Human leukocyte antigens; MNCs: Mononuclear cells; MSCs: Multipotent mesenchymal stromal/stem cells; BM: Bone marrow; FBS: Fetal bovine serum;

EDTA: Ethylenediaminetetraacetic acid; ORO: Oil Red O; ARS: Alizarin Red S; bFGF: Basic fibroblast growth factor; DPBS: Dulbecco's phosphate-buffered saline; BSA: Bovine serum albumin; DAPI: Diamidino-phenylindole; STF: SCF, TPO, Flt3-ligand; BMP4: Bone morphogenic protein 4; ATRA: All-trans retinoic acid

\section{Supplementary Information}

The online version contains supplementary material available at https://doi. org/10.1186/s13287-021-02364-z.

Additional file 1: Figure S1. Karyotype analysis of healthy donor and GS-2 iPSC clones. For karyotyping, iPSC cultures were trypsinized and treated with a hypotonic salt solution. 10 metaphases were captured and analysed. Shown here are the karyotypes of a healthy donor (left, GP) and the 3 GS-2 patient-derived iPSCS (IK, YF, YKÇ). Figure S2. Upper panel: iPSCs were co-cultured with Op9 cells in presence of HDM2 medium (StemMACS HSC expansion medium, 1X STF, $10 \mathrm{\mu g} / \mathrm{mL}$ rhBMP-4), resulting in low level expression of CD34. Lower panel: further expansion of iPSC-derived HSCs in StemMACS HSC expansion medium, 1X STF for 21 days resulted in expansion of CD43+, CD34+, CD45+ HSCs. Table S1. Primer sequences used for RT-PCR. Table S2. Differentiation capacity of healthy donor and GS-2 BM-MSCs. For adipogenic differentiation, MSCS were cultured in DMEM-LG, supplemented with 10\% FBS, 1 MM dexamethasone, $60 \mu \mathrm{M}$ indometacin, $500 \mu \mathrm{M}$ 3-isobutyl-1-methylxanthine and $5 \mu \mathrm{g} / \mathrm{mL}$ insulin. After 3 weeks, cells were stained with $2 \mathrm{mg} / \mathrm{mL}$ Oil Red O (ORO, Sigma-Aldrich O0625). ORO dye was extracted from the cells using \%2 lgepal and measured at $496 \mathrm{~nm}$ on a microplate reader. For osteogenic differentiation, MSCs were cultured in DMEM-LG, 10\% FBS, $100 \mathrm{nM}$ dexamethasone, $10 \mathrm{mM}$ beta-glycerophosphate and $0,2 \mathrm{mM} \mathrm{L}$ ascorbic acid. Calcium levels were measured using the Quantichrom Calcium Analysis kit (BioAssay Systems, DICA-500). Table S3. Immunophenotype of healthy donor and GS-2 BM-MSCs. MSCs from healthy donors and GS-2 patients were generally positive for MSC-specific surface antigens (CD29, CD44, CD73, CD90, CD105 and CD166) and negative for hematopoietic and endothelial cell markers. No significant differences were found in expression of cell surface antigens by MSCs between the healthy donors and GS-2 patients.

\section{Acknowledgements}

Not applicable.

\section{Authors' contributions}

GGE performed the experiments, was involved in the data acquisition and data analysis, and drafted the manuscript. ÖDE, BP, GGS, and EB were involved in the data acquisition and/or analysis. TO, PK, AGO, and DUÇ helped with the interpretation of the data. FAK designed the study, acquired funding, and interpreted the data. The authors read and approved the final manuscript.

\section{Funding}

This study was supported by a grant from the Scientific and Technological Research Council of Turkey (TUBITAK) project nr 2145071.

\section{Availability of data and materials}

Data are made available through supplementary materials. Data not available through the supplementary materials are available from the corresponding author on reasonable request.

\section{Declarations}

\section{Ethics approval and consent to participate}

Procedures and sampling were approved by the Hacettepe University NonInterventional Ethical Committee (GO14/424-19). All samples were obtained after informed consent. All animal experiments were approved by the local Hacettepe University animal experiments ethical committee (2014/46-03 and 2015/68-10) and performed at the Hacettepe University Laboratory Animals Research and Application Center.

\section{Consent for publication}

Consent for publication was obtained from the parents of the individuals involved. There are no patient-specific data provided in this manuscript.

\section{Competing interests}

The authors declare that they have no competing interests.

\section{Author details}

${ }^{1}$ Graduate School of Health Sciences, Department of Stem Cell Sciences, Hacettepe University, Sinhiye, 06100 Ankara, Turkey. ${ }^{2}$ Center for Stem Cell Research and Development (PEDI-STEM), Hacettepe University, Sihhiye, 06100 Ankara, Turkey. ${ }^{3}$ School of Medicine, Research Center for Translational Medicine, Koç University, Istanbul, Turkey. ${ }^{4}$ Faculty of Medicine, Department of Histology and Embryology, Hacettepe University, Sihhiye, 06100 Ankara, Turkey. ${ }^{5}$ Faculty of Medicine, Department of Pediatrics, Division of Hematology, Hacettepe University, Ankara, Turkey. ${ }^{6}$ Laboratory Animals Research and Application Center (HUDHAM), Hacettepe University, Sinhiye, 06100 Ankara, Turkey.

Received: 1 April 2021 Accepted: 29 April 2021

Published online: 13 May 2021

\section{References}

1. Kuskonmaz B, Ayvaz D, Gokce M, Ozgur TT, Okur FV, Cetin M, et al. Hematopoietic stem cell transplantation in children with Griscelli syndrome: a single-center experience. Pediatr Transplant. 2017;21(7):e13040, 1-7. https://doi.org/10.1111/petr.13040.

2. Singh A, Garg A, Kapoor S, Khurana N, Entesarian M, Tesi B. An Indian boy with Griscelli syndrome type 2: case report and review of literature. Indian J Dermatol. 2014;59(4):394-7. https://doi.org/10.4103/0019-5154.135494.

3. Menasche G, Pastural E, Feldmann J, Certain S, Ersoy F, Dupuis S, et al. Mutations in RAB27A cause Griscelli syndrome associated with haemophagocytic syndrome. Nat Genet. 2000;25(2):173-6. https://doi.org/1 $0.1038 / 76024$.

4. Meschede IP, Santos TO, Izidoro-Toledo TC, Gurgel-Gianetti J, Espreafico EM. Griscelli syndrome-type 2 in twin siblings: case report and update on RAB27A human mutations and gene structure. Braz I Med Biol Res. 2008; 41(10):839-48. https://doi.org/10.1590/50100-879X2008001000002.

5. Meeths M, Bryceson YT, Rudd E, Zheng C, Wood SM, Ramme K, et al. Clinical presentation of Griscelli syndrome type 2 and spectrum of RAB27A mutations. Pediatr Blood Cancer. 2010;54(4):563-72. https://doi.org/10.1002/ pbc.22357.

6. Al-Ahmari A, Al-Ghonaium A, Al-Mansoori M, Hawwari A, Eldali A, Ayas M, et al. Hematopoietic SCT in children with Griscelli syndrome: a single-center experience. Bone Marrow Transplant. 2010;45(8):1294-9. https://doi.org/10.1 038/bmt.2009.358.

7. Arico M, Zecca M, Santoro N, Caselli D, Maccario R, Danesino C, et al. Successful treatment of Griscelli syndrome with unrelated donor allogeneic hematopoietic stem cell transplantation. Bone Marrow Transplant. 2002; 29(12):995-8. https://doi.org/10.1038/sj.bmt.1703567.

8. Baumeister FA, Stachel D, Schuster F, Schmid I, Schaller M, Wolff H, et al. Accelerated phase in partial albinism with immunodeficiency (Griscelli syndrome): genetics and stem cell transplantation in a 2-month-old girl. Eur J Pediatr. 2000:159(1-2):74-8. https://doi.org/10.1007/PL00013808.

9. Takahashi K, Tanabe K, Ohnuki M, Narita M, Ichisaka T, Tomoda K, et al. Induction of pluripotent stem cells from adult human fibroblasts by defined factors. Cell. 2007;131(5):861-72. https://doi.org/10.1016/j.cell.2007.11.019.

10. Georgomanoli M, Papapetrou EP. Modeling blood diseases with human induced pluripotent stem cells. Dis Model Mech. 2019;12(6):dmm039321, 120. https://doi.org/10.1242/dmm.039321.

11. Polo JM, Liu S, Figueroa ME, Kulalert W, Eminli S, Tan KY, et al. Cell type of origin influences the molecular and functional properties of mouse induced pluripotent stem cells. Nat Biotechnol. 2010;28(8):848-55. https://doi.org/1 $0.1038 / \mathrm{nbt} .1667$

12. Casey J. Modeling pathology with IPS cells. Nature Reviews Neurol. 2009; 5(4):180. 
13. Morrison M. Infrastructural expectations: exploring the promise of international large-scale induced pluripotent stem cell banks. New Genetics Soc. 2017;36(1):66-83. https://doi.org/10.1080/14636778.2017.1289470.

14. De Sousa PA, Steeg R, Wachter E, Bruce K, King J, Hoeve M, et al. Rapid establishment of the European Bank for induced Pluripotent Stem Cells (EBiSC) - the hot start experience. Stem Cell Research. 2017;20:105-14. https://doi.org/10.1016/j.scr.2017.03.002.

15. McKernan R, Watt FM. What is the point of large-scale collections of human induced pluripotent stem cells? Nat Biotechnol. 2013;31(10):875-7. https:// doi.org/10.1038/nbt.2710.

16. Uckan D, Kilic E, Sharafi P, Kazik M, Kaya F, Erdemli E, et al. Adipocyte differentiation defect in mesenchymal stromal cells of patients with malignant infantile osteopetrosis. Cytotherapy. 2009;11(4):392-402. https:// doi.org/10.1080/14653240802582083.

17. Warlich E, Kuehle J, Cantz T, Brugman MH, Maetzig T, Galla M, et al. Lentiviral vector design and imaging approaches to visualize the early stages of cellular reprogramming. Mol Ther. 2011;19(4):782-9. https://doi. org/10.1038/mt.2010.314.

18. Livak KJ, Schmittgen TD. Analysis of relative gene expression data using real-time quantitative PCR and the $2^{-\Delta \Delta C T}$ method. Methods (San Diego, Calif). 2001;25(4):402-8

19. van Til NP, de Boer H, Mashamba N, Wabik A, Huston M, Visser TP, et al. Correction of murine Rag2 severe combined immunodeficiency by lentiviral gene therapy using a codon-optimized RAG2 therapeutic transgene. Mol Ther. 2012;20(10):1968-80. https://doi.org/10.1038/mt.2012.110.

20. Holmes R, Zuniga-Pflucker JC. The OP9-DL1 system: generation of Tlymphocytes from embryonic or hematopoietic stem cells in vitro. Cold Spring Harb Protoc. 2009;2009(2):pdb prot5156.

21. Vodyanik MA, Thomson JA, Slukvin II. Leukosialin (CD43) defines hematopoietic progenitors in human embryonic stem cell differentiation cultures. Blood. 2006;108(6):2095-105. https://doi.org/10.1182/blood-200602-003327.

22. Haddad EK, Wu X, Hammer JA 3rd, Henkart PA. Defective granule exocytosis in Rab27a-deficient lymphocytes from Ashen mice. J Cell Biol. 2001;152(4): 835-42. https://doi.org/10.1083/jcb.152.4.835.

23. Pachlopnik Schmid J, Ho CH, Diana J, Pivert G, Lehuen A, Geissmann F, et al. A Griscelli syndrome type 2 murine model of hemophagocytic lymphohistiocytosis (HLH). Eur J Immunol. 2008;38(11):3219-25. https://doi. org/10.1002/eji.200838488.

24. Abyzov A, Mariani J, Palejev D, Zhang Y, Haney MS, Tomasini L, et al. Somatic copy number mosaicism in human skin revealed by induced pluripotent stem cells. Nature. 2012;492(7429):438-42. https://doi.org/10.103 8/nature11629.

25. Wang J, Gu Q, Hao J, Bai D, Liu L, Zhao X, et al. Generation of induced pluripotent stem cells with high efficiency from human umbilical cord blood mononuclear cells. Genomics Proteomics Bioinformatics. 2013;11(5): 304-11. https://doi.org/10.1016/j.gpb.2013.08.002.

26. Noguchi H, Miyagi-Shiohira C, Nakashima Y. Induced tissue-specific stem cells and epigenetic memory in induced pluripotent stem cells. Int J Mol Sci. 2018;19(4):930, 1-11. https://doi.org/10.3390/ijms19040930.

27. Kim K, Doi A, Wen B, Ng K, Zhao R, Cahan P, et al. Epigenetic memory in induced pluripotent stem cells. Nature. 2010;467(7313):285-90. https://doi. org/10.1038/nature09342.

28. Choi KD, Yu J, Smuga-Otto K, Salvagiotto G, Rehrauer W, Vodyanik M, et al. Hematopoietic and endothelial differentiation of human induced pluripotent stem cells. Stem Cells. 2009;27(3):559-67. https://doi.org/10.1 002/stem.20080922.

29. Chute JP, Muramoto GG, Whitesides J, Colvin M, Safi R, Chao NJ, et al. Inhibition of aldehyde dehydrogenase and retinoid signaling induces the expansion of human hematopoietic stem cells. Proc Natl Acad Sci U S A. 2006;103(31):11707-12. https://doi.org/10.1073/pnas.0603806103.

30. D'Souza SS, Maufort J, Kumar A, Zhang J, Smuga-Otto K, Thomson JA, et al. GSK3 $\beta$ inhibition promotes efficient myeloid and lymphoid hematopoiesis from non-human primate-induced pluripotent stem cells. Stem Cell Reports. 2016;6(2):243-56. https://doi.org/10.1016/j.stemcr.2015.12.010.

31. Galat Y, Dambaeva S, Elcheva I, Khanolkar A, Beaman K, lannaccone PM, et al. Cytokine-free directed differentiation of human pluripotent stem cells efficiently produces hemogenic endothelium with lymphoid potential. Stem Cell Res Ther. 2017;8(1):67. https://doi.org/10.1186/s13287-017-0519-0.
32. Huber TL, Zhou Y, Mead PE, Zon LI. Cooperative effects of growth factors involved in the induction of hematopoietic mesoderm. Blood. 1998;92(11): 4128-37. https://doi.org/10.1182/blood.V92.11.4128.

33. Cerdan $\mathrm{C}$, Hong $\mathrm{SH}$, Bhatia M. Formation and hematopoietic differentiation of human embryoid bodies by suspension and hanging drop cultures. Curr Protoc Stem Cell Biol. 2007;Chapter 1:Unit 1D 2.

34. Beyer Al, Muench MO. Comparison of human hematopoietic reconstitution in different strains of immunodeficient mice. Stem Cells Dev. 2017;26(2): 102-12. https://doi.org/10.1089/scd.2016.0083.

\section{Publisher's Note}

Springer Nature remains neutral with regard to jurisdictional claims in published maps and institutional affiliations.

\section{Ready to submit your research? Choose BMC and benefit from:}

- fast, convenient online submission

- thorough peer review by experienced researchers in your field

- rapid publication on acceptance

- support for research data, including large and complex data types

- gold Open Access which fosters wider collaboration and increased citations

- maximum visibility for your research: over $100 \mathrm{M}$ website views per year

At BMC, research is always in progress.

Learn more biomedcentral.com/submissions 